\title{
Mapping of the turbulent round jet developing region using a constant temperature anemometer (CTA)
}

\author{
Mohd Rusdy Yaacob ${ }^{a,}$, , Rasmus Korslund Schlander ${ }^{b}$, Preben Buchhave ${ }^{c}$, Clara Marika Velte ${ }^{b}$ \\ a Faculty of Electrical Engineering, Universiti Teknikal Malaysia Melaka, Durian Tunggal, Malaysia \\ ${ }^{b}$ Department of Mechanical Engineering, Technical University of Denmark, Lyngby, Denmark \\ c Intarsia Optics, Sønderskovvej 3, Birkerød, Denmark
}

* Corresponding author: rusdy@utem.edu.my

\section{Article history}

Received 3 May 2018

Revised 25 August 2018

Accepted 11 September 2018

Published Online 25 October 2018

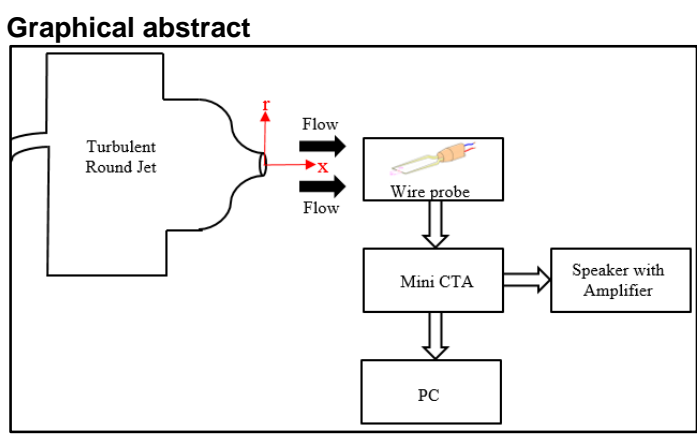

\begin{abstract}
The fully developed round turbulent jet has been extensively studied, whereas the developing region is much less understood. The high shear and turbulence intensities in the most interesting parts of the developing region make them inaccessible to common measurement techniques such as Constant Temperature Anemometry (CTA) due to the high demands on the measurement techniques for accuracy of the measurements. Turbulence measurements are therefore planned using our in-house laser Doppler anemometer (LDA) system based on its capability to provide accurate measurements and with its inherent ability to properly distinguish velocity components. A rigorous measurement with the intended LDA system however demands impractical processing time, so knowing the critical points at which measurement are to be taken will save valuable time. This information is herein acquired significantly faster and more practically, however less accurately, with single-wire CTA. A high-resolution measurement was done using a computer-controlled single-wire CTA with the wire probe mounted perpendicular to the incoming flow from the jet orifice. The measurements covered several points in the radial ( $r$-direction) along $x / D=10, x / D=15$, $x / D=20$ and $x / D=30$ downstream (where $D$ is the jet exit diameter), with spatia resolutions ranging from 1 to $3 \mathrm{~mm}$ between the points, depending on how far the measurement was from the jet centerline. A proper alignment was also conducted prior to measurement so that the same points can be reached again for LDA measurement on the same jet afterwards. The radial profiles of mean velocity and turbulence intensity at each downstream position are presented to show the statistics of the air flow inside and outside the jet. As expected from theory, the mean profiles display a nearly Gaussian shape, spread out and tapered with the downstream direction. The highest velocities are located at the centerline.
\end{abstract}

Keywords: Hot wire; turbulent round jet, turbulence

\section{INTRODUCTION}

The flow field of a turbulent round jet is not only divided into the delevoping and fully developed regions in the streamwise direction (Mossa \& Serio, 2016), but also in the variations along the radial direction which comprises three different layers viz. centreline layer, shear layer region and outer region (Ball et al., 2012). These regions and layers are clearly illustrated in Fig. 1 and Fig. 2. The turbulent round jet is a classical turbulent flow of special interest for fundamental investigations (Hinze, 1975), where the fully developed region has been extensively studied, see e.g. (Hussein et al., 1994). Due to the difficulties in measuring accurately high shear and high turbulence intensity flows, the developing region of the jet has remained substantially underexplored. This developing region is particularly interesting, however, since it allows for studying the downstream (or temporal) development of the turbulence cascade from non-equilibrium to an equilibrium state (in the fully developed jet). Accurate measurements in this region may thus provide highly valuable and novel information that can challenge the established theory of turbulence (Vassilicos, 2015).

LDA is capable of acquiring velocity measurements nonintrusively (i.e. without disturbing the flow) (Buchhave et al., 1979) and are also able to accurately distinguish the spatial velocity components, even at high turbulence intensities (Hussein et al., 1994). However, our improved in-house LDA system (Velte et al., 2017; Buchhave \& Velte, 2017; M. R. Yaacob et al., 2018) demands much longer computational time compared to computer-controlled CTA, which is faster and easier to use especially with automated calibration (Jørgensen, 1996). Having to obtain the mean velocity and turbulence intensity profiles quickly from CTA measurement in our underinvestigation turbulent round jet, will be time-efficient and the results will be useful for the upcoming high-resolution LDA measurement in mapping the developing region of the same jet using the same flow conditions. The results are aimed to help us in finding the boundary between the jet and the surrounding air, and also the shear layer for different downstream positions. 


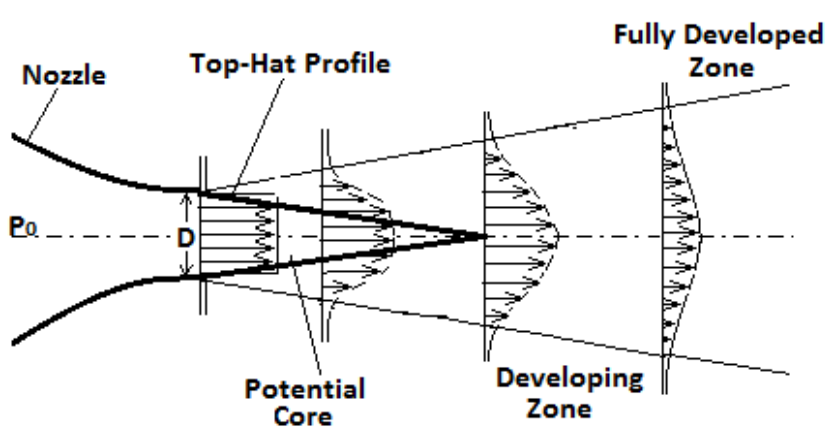

Fig. 1 Streamwise development and the various regions of a turbulent round jet (Kaushik et al., 2015).

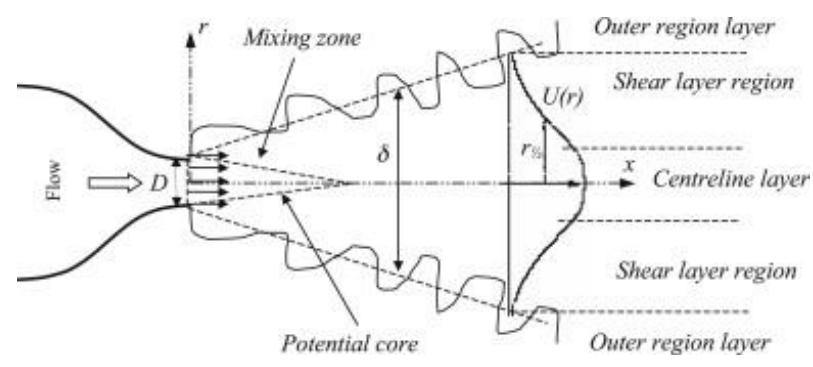

Fig. 2 Different layers of turbulent round jet in radial direction (Fellouah et al., 2009).

\section{EXPERIMENTAL METHOD}

CTA provides velocity time-history information in one measurement position. Based on the convective heat transfer principle (Jørgensen, 2002), the passing air will cause temperature change on the heated wire placed within the flow. The anemometer is keeping the wire at a constant temperature by adjusting the voltage passing through the wire as depicted in Fig. 3.

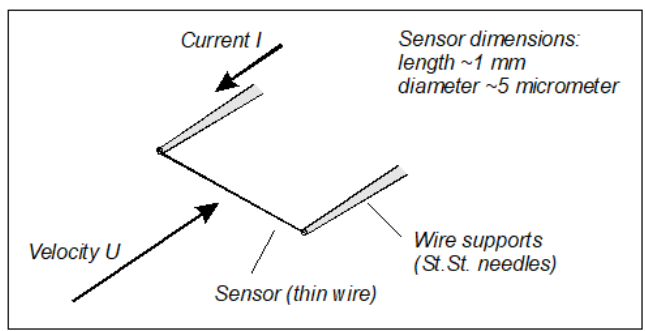

Fig. 3 Close-up of support and wire-sensor with a velocity, $U$, and current, I in the wire (F. Gökhan Ergin, 2016).

The jet box used for these investigations was made of aluminum with dimensions $58 \times 58.5 \times 59 \mathrm{~cm}$ and fitted with an outer nozzle which was designed to condition the flow to follow as closely as possible a laminar top-hat profile at the jet exit with diameter $D=10$ $\mathrm{mm}$. The turbulent round jet itself was a replica of the one used by (Velte et al., 2014), from which further details can be found. The jet input pressure was set to 1 bar, corresponding to a jet exit velocity $\approx 30$ $\mathrm{m} / \mathrm{s}$ and Reynolds number, $R e \approx 20,000$.

Prior to the measurements, the system was first calibrated using an automatic calibration system from Dantec Dynamics. This was separately done by increasing the air flow in accordance with pressure difference inside a well conditioned wind tunnel and then fitting a 4thorder polynomial to a relation between output voltage and velocity. The experimental settings listed in Table 1 were considered during the calibration.
Table 1 Experiment setting.

\begin{tabular}{ll}
\hline Sampling frequency & $10 \mathrm{kHz}$ \\
Sampling time & $10 \mathrm{~s}$ \\
Number of samples & $100 \mathrm{kS}$ \\
Temperature & $\approx 21.5^{\circ} \mathrm{C}$ \\
Atmospheric pressure & $\approx 1015 \mathrm{hPa}$ \\
\hline
\end{tabular}

The measurements were conducted using a uni-directional single sensor probe where the thin wire was placed perpendicular to the incoming flow from the jet orifice. The probe was mounted on a support (see Fig. 4) and connected to the CTA anemometer and computer installed with Dantec Dynamics MiniCTA software (see Fig. 5), from which a single time series data was reduced to a series of fluctuating velocity of the streamwise component, leading to the calculation of mean and rms velocity for each measurement point.

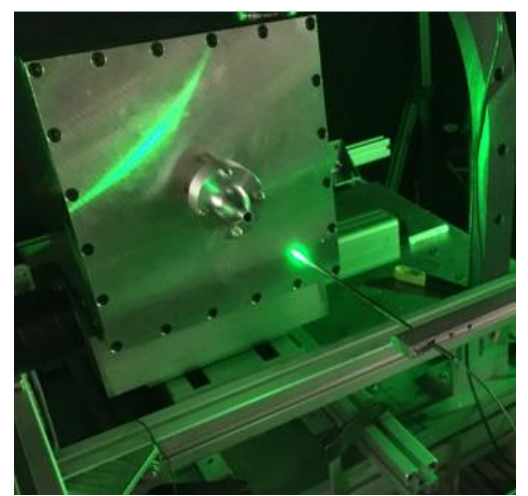

Fig. 4 CTA experiment showing jet orifice and the support mounted wire probe.

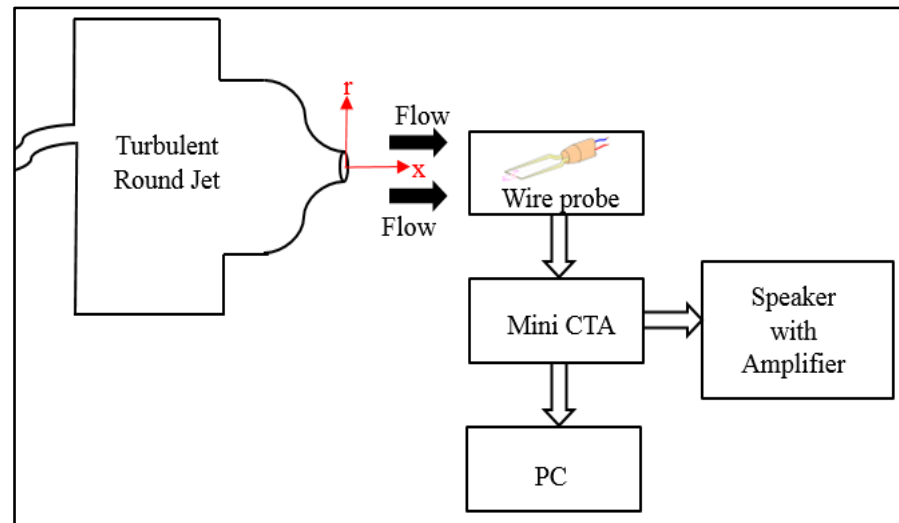

Fig. 5 Overall experimental setup. A speaker with amplifier was also installed at the CTA output to produce noise from the turbulence to help locating regions of various turbulence intensity such as the highest shear layer.

The measurement covered several points in the radial ( $r$-direction) along $x / D=10, x / D=15, x / D=20$ and $x / D=30$ downstream, with resolution ranging from 1 to $3 \mathrm{~mm}$ between the points, depending on how far the measurements were from the jet centreline. The jet box was mounted on a 3-axis traversing system so that it can be easily manoeuvred to the desired points correspondingly when performing the LDA measurement.

Having in mind that the results will map the points or region of interest for the incoming LDA measurement, the probe was first carefully aligned (see Fig. 6) so that the thin wire was placed at the pinhole of a photodetector (M.R. Yaacob et al., 2018), at which the jet centreline and measurement volume of LDA has been identified to overlap beforehand, without moving or changing the position of the photodetector. This location of the probe was fixed while traversing only the jet throughout the CTA measurement. The same points can therefore consequently be reached again for LDA measurement. 


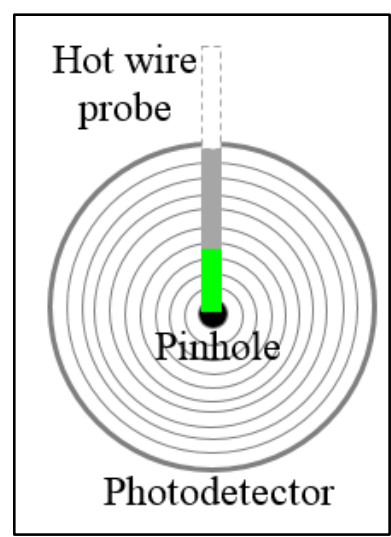

Fig. 6 View through a photodetector showing probe alignment with the pinhole.

\section{RESULTS AND DISCUSSION}

In the figures presented herein, the data have not been normalized or fitted to any order of polynomial, in order to display the direct flow results. Fig. 7 shows the radial profiles of mean velocity at various downstream positions throughout the developing region, namely $x / D=$ $10,15,20 \& 30$. As expected from theory, the profiles seem to follow a nearly Gaussian shape, spread out and tapered with the downstream position. The highest average velocities are spotted at the centreline, while the points where average velocity is very close to zero indicate that the measurement was somewhere near the boundary between the jet and the surrounding air, which should be noted when conducting the LDA measurement.

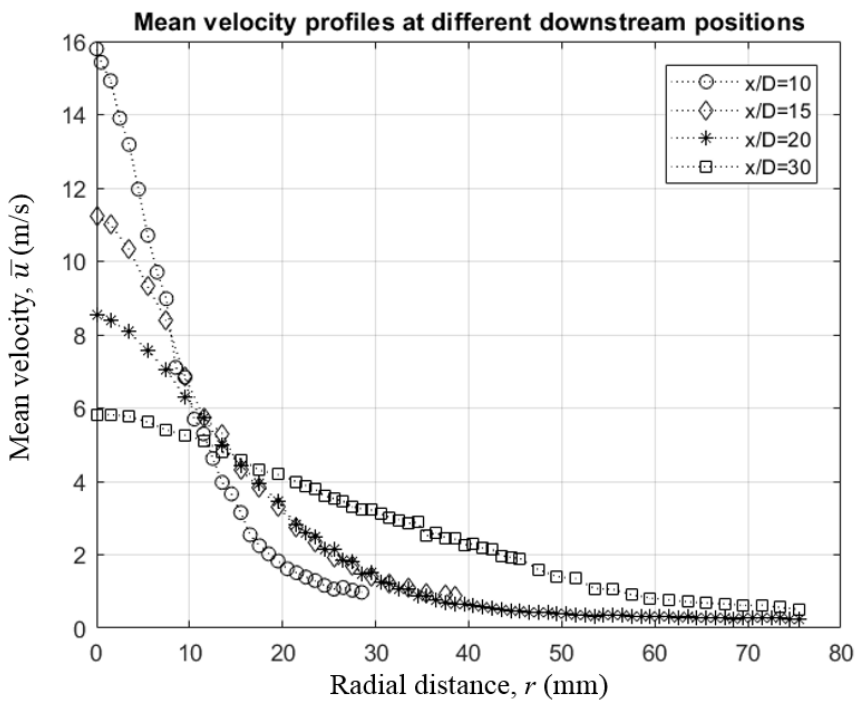

Fig. 7 Radial profiles of mean velocity at different downstream positions $x / D=10,15,20 \& 30$ where $D$ is the jet exit diameter.

Fig. 8 shows radial profiles of the turbulence intensity, $I$, at different downstream positions:

$I=\sqrt{\frac{\overline{u^{\prime 2}}}{\bar{u}^{2}}}$

where $\bar{u}$ is the mean velocity and $u^{\prime}$ is the rms velocity.

When the measurements progress away from the centerline, the turbulence intensity increases in the region where the shear layer is located. In these regions, the high levels of fluctuations will require an appropriate frequency shift to measure accurately with the LDA system (Buchhave, 1984). Again, keep in mind that the profiles obtained are just an approximation (and intended only to map the measurement points for the latter LDA measurement) and does not accurately describe turbulence especially in the region where fluctuations are large compared to the streamwise convection. More accurate counterparts measured using LDA, showing a rapid growth of turbulence intensity with radial distance from the centerline, can be found in (Buchhave \& Velte, 2017). However the approximation is still more acceptable when turbulence intensities are low as the streamwise convection dominates over the fluctuations.

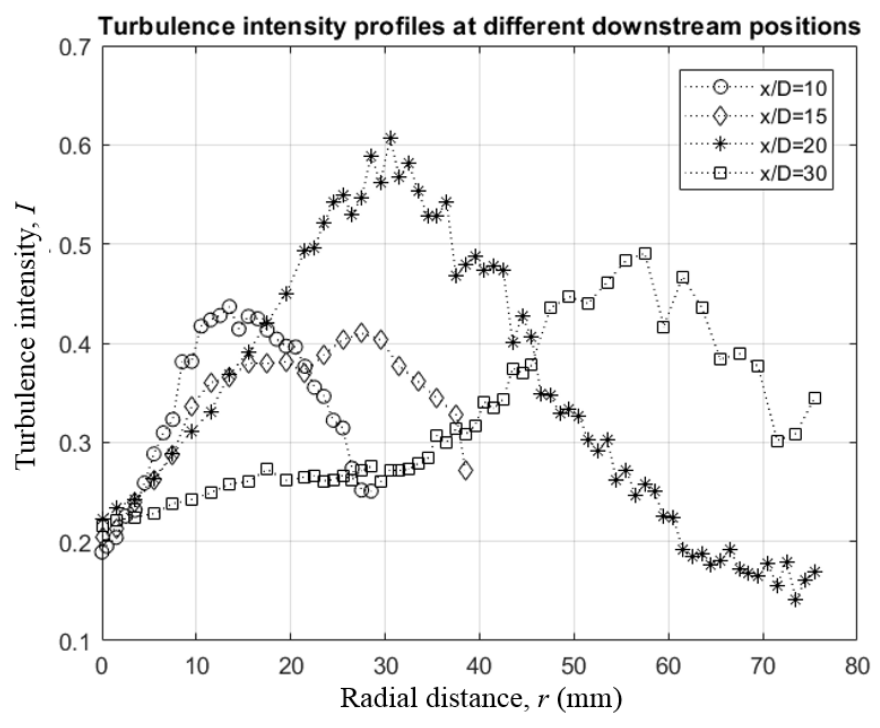

Fig. 8 Radial profiles of streamwise turbulence intensity at different downstream positions $x / D=10,15,20 \& 30$ where $D$ is the jet exit diameter. With more accurate LDA measurements, the turbulence intensity can be seen to monotonically increase rapidly with radial distance (Buchhave \& Velte, 2017).

\section{CONCLUSION}

A CTA measurement was successfully performed to provide a measurement scheme and to identify the spatial extent of regions of interest for future, more rigorous LDA turbulence measurement. The outcome of the planned LDA measurements will be published in a separate scientific article.

\section{ACKNOWLEDGEMENT}

The authors wish to acknowledge the support of Ministry of Higher Education Malaysia, Reinholdt W. Jorck og Hustrus Fond (grant journal no. 13-J9-0026) and Fabriksejer, Civilingeniør Louis Dreyer Myhrwold og hustru Janne Myhrwolds Fond (grant journal no. 13-M70039 and 15-M7-0031). Also we are grateful to Benny Edelsten (Laboratory Engineer) for helping out during the measurement.

\section{REFERENCES}

Ball, C. G., Fellouah, H. \& Pollard, A., 2012. The flow field in turbulent round free jets. Progress in Aerospace Sciences, 50, pp. 1-26.

Buchhave, P., 1984. Three-component LDA measurements. Disa Information, (29), pp. 3-9.

Buchhave, P., George, W. K. \& Lumley, J. L., 1979. The measurement of turbulence with the laser-Doppler anemometer. Annual Review of Fluid Mechanics, 11, pp. 443-503.

Buchhave, P. \& Velte, C. M., 2017. Measurement of turbulent spatial structure and kinetic energy spectrum by exact temporal-to-spatial mapping. Physics of Fluids, 29(8), 085109.

F. Gökhan Ergin, 2016. Constant Temperature Anemometry - A Theoretical Introduction. Dantec Dynamics.

Fellouah, H., Ball, C. G. \& Pollard, A., 2009. Reynolds number effects within the development region of a turbulent round free jet. International Journal of Heat and Mass Transfer, 52(17-18), pp. 3943-3954.

Hinze, J. O., 1975. Turbulence 2nd ed., Michigan: McGraw-Hill.

Hussein, H. J., Capp, S. P. \& George, W. K., 1994. Velocity measurements in a high-Reynolds-number, momentum-conserving, axisymmetric, turbulent jet. Journal of Fluid Mechanics, 258, pp. 31-75. 
Jørgensen, F. E., 2002. How to measure turbulence with hot-wire anemometers - A practical guide. Dantec Dynamics.

Jørgensen, F. E., 1996. The computer-controlled Aspects of set-up, probe calibration, data acquisition and data conversion. Measurement Science and Technology, 7(10), pp.1378-1387.

Kaushik, M., Kumar, R. \& Humrutha, G., 2015. Review of computational fluid dynamics studies on jets. American Journal of Fluid Dynamics, 5(3A), pp.1-11.

Mossa, M. \& Serio, F. De, 2016. Rethinking the process of detrainment : Jets in obstructed natural flows. Bari: Nature Publishing Group.

Vassilicos, J. C., 2015. Dissipation in turbulent flows. Annual Review of Fluid Mechanics, 47, pp. 95-114.

Velte, C. M., Buchhave, P. \& Hodzic, A., 2017. Measurement of turbulent kinetic energy spectrum - Part 2 : Convection record measurements. In $i T i$ Conference on Turbulence VII. Springer, pp. 171-176.

Velte, C. M., George, W. K. \& Buchhave, P., 2014. Estimation of burst-mode LDA power spectra. Experiments in Fluids, 55(1674), p. 1-20.

Yaacob, M. R., Schlander, R. K., Velte, C. M., \& Buchhave, P., 2018. Validation of improved laser Doppler anemometer (LDA) based on the fully developed turbulent round jet. Symposium of Electrical, Mechatronics and Applied Science. November 2018. Melaka. [Manuscript accepted for publication].

Yaacob, M. R., Schlander, R. K., Velte, C. M., \& Buchhave, P., 2018 Experimental evaluation of kolmogorov's $-5 / 3$ and 2/3 power laws in the developing turbulent round jet. Journal of Advanced Research in Fluid Mechanics and Thermal Sciences, 45(2), pp. 14-21. 